\title{
Efficient Image Transmission System Using IFS
}

\author{
Sang Hyun Kim ${ }^{1 *}$ \\ ${ }^{1}$ Department of Electronic Engineering, Kyungpook National University \\ IFS를 이용한 고효율 영상전송 시스템 \\ 김상현:* \\ ${ }^{1}$ 경북대학교 산업전자공학과
}

\begin{abstract}
The concept of IFS (Iterated Function System) was applied to compress and transmit image data efficiently. To compress the image data with IFS, self-similarity was used to search a similar block. To improve the coding performance for the iterated function system with natural images, the image will be formed of properly transformed parts of itself to minimize the coding error. The simulation results using the proposed IFS represent high PSNR performance and improved compression efficiency with the coefficient of a recursive function.

요 약 본 논문에서는 영상 데이터를 효율적으로 압축하고 전송하기 위해 IFS (반복함수시스템) 개념을 적용하였다. 영상 데이터 압축에 IFS를 적용하기 위해 자기유사도를 사용하여 동일한 영상에서 코딩하고자 하는 블록과 유사한 부분을 찾게 된다. 실제 영상에 대한 반복함수시스템 코딩의 정확도 향상을 위해서 영상의 각 세부 블록에 대해 에러를 최소화 하는 변환 블록을 적용하여 성능을 높였다. 제안한 IFS를 사용한 실험결과에서 높은 화질성능을 보였고 반복함수 계수만을 압축된 영상데이터로 사용함으로써 압축효율을 향상시킬 수 있다.
\end{abstract}

Key Words : IFS (iterated function system), image transmission, block coding

\section{Introduction}

The IFS coding consists of a description of both an image partition and a contractive image transformation defined as a list of parent and child block transformations, each specified by a small set of quantized parameters [1]. IFS has the intrinsic property of having extremely high visual complexity while being very low in information content, as they can be described and generated by simple recursive deterministic algorithms. They are mathematical objects with a high degree of redundancy in the sense that they are recursively made of transformed copies of either themselves or parts of themselves. The IFS has also been part of a set of tools in a variety of fields in physics, where they are closely related to Chaos theory. IFS based techniques have been applied in several areas of digital image processing [2]. The mathematical theories of IFS constitute the broad foundations of image compression. Conventional algorithm using IFS has huge computational complexity whereas we are concerned with fully automated robust, block-based IFS image coding schemes which can compress and encode any digital image efficiently.

\section{IFS Image Generation}

This work was supported by the Kyungpook National University Research Grant, 2011.

${ }^{*}$ Corresponding Author : Sang Hyun Kim(Kyungpook National Univ.)

Tel: +82-10-2702-4138 email: shk@knu.ac.kr 
IFS image can be generated with IFS parameters using affine transforms. Steps to generate IFS image follows:

Step 1) Start with a piece of blank paper with an $(\mathrm{x}, \mathrm{y})$ coordinate system marked and pick an arbitrary on the paper, then find its coordinates.

Step 2) Randomly select one of the four affine transforms listed blows:

$$
\begin{aligned}
& \text { W1: }(\mathrm{x}, \mathrm{y})->(0.49 \mathrm{x}+0.01 \mathrm{y}+25,0.62 \mathrm{y}-2), \\
& \text { W2: }(\mathrm{x}, \mathrm{y})->(0.27 \mathrm{x}+0.52 \mathrm{y},-0.4 \mathrm{x}+0.36 \mathrm{y}-56), \\
& \text { W3: }(\mathrm{x}, \mathrm{y})->(0.18 \mathrm{x}-0.73 \mathrm{y}+88,0.5 \mathrm{x}+0.26 \mathrm{y}+8), \\
& \text { W4: }(\mathrm{x}, \mathrm{y})->(0.4 \mathrm{x}-0.01 \mathrm{y}+52,0.5 \mathrm{x}+32)
\end{aligned}
$$

Step 3) Apply the transform to this point, and the coordinates of a new point are obtained. Again select randomly one of above four transforms and apply it to the point to obtain the next new point. These four affine transforms form an iterated function system.

Different transformations lead to different attractors, with the technical limitation that the transformations must be contractive. That is, a given transformation applied to any two points in the input image must bring them closer together. Generated images with IFS are shown in [Fig. 1]. An image with IFS can be completely described by mathmatical algorithm in its infinitely fine texture and detail.

\section{IFS Image Coding}

The three main issues involved in the design of IFS are the partitioning of an image, the choice of a measure of distortion between two images, and the specification of a finite class of contractive image transformations defined consistently with a partition, and of a scheme for the quantization of their parameters.

\section{A. Distortion Measure}

The square support of the original digital image is

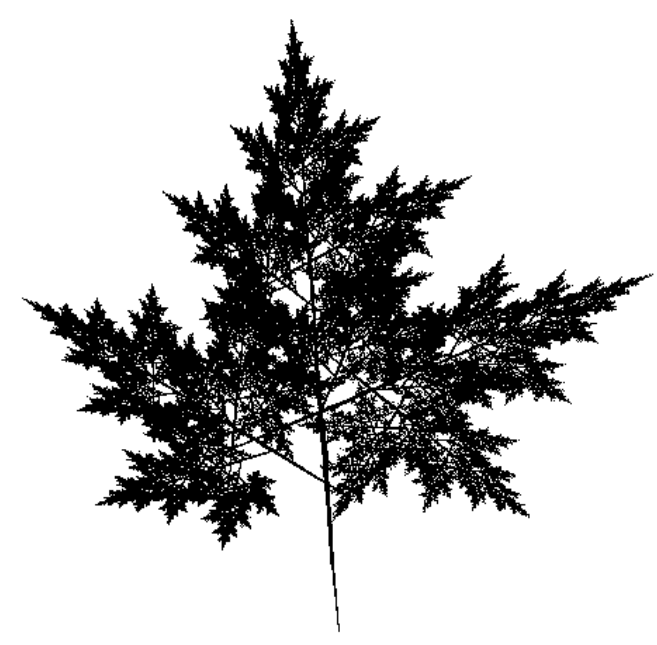

[Fig. 1] IFS image generation (iteration number $=2,000,000$ )

partitioned into non overlapping square range cells of two different sizes, thus forming a two-level square partition. A parent can be split into up to four non-overlapping children.

Decisions about the splitting of a parent cell are made during the encoding of the image block over this cell. A partition constructed in this way is image-dependent. The selection of sizes and shapes for image cells depends on several factors. Small image blocks are easy to analyze and to classify geometrically. They allow a fast evaluation of interblock distances and they are easy to encode accurately [3].

We construct a distortion measure between digital images from an interblock distortion measure. The root-mean-square distortion between the image blocks is defined as the square root of the sum over the cell. We define the distance between two images as the sum over the partition of all block distortions.

\section{B. IFS codes}

An original image quantized 256 gray levels is given as input to the coder. Given a reference block $R$, (of size B X B), the construction of a transformation which maps onto this cell is broken into two distinct steps corresponding to the transformations. 
The construction of the spatial contraction amounts to selecting an image domain block of size D X D, which will be contracted to a block of size B X B. It is important to note that the specification of the domain cell $D$ (location and size) is equivalent to the description of the spatial contraction (translation vector and contraction factor). The second part of the construction consists of selecting the proper processing of the contracted domain block. We can thus formally define a pool of domain blocks $D$, made up of all image blocks which can be extracted from the original image, and which are larger than the reference block $R$. We can also formally define a pool of transformations, made of all discrete block transformations. The encoding of the reference block consists in finding a best pair, which has a minimum distortion.

For input image frame dissimilarity is evaluated with directed divergence measure

$$
F(p, q)=A \int q(x) \log \frac{q(x)}{p(x)} d x+B \int p(x) \log \frac{p(x)}{q(x)} d x
$$

using the histogram between interframe. For interframe pair with large directed divergence temporal block matching is employed. In case of similar interframe efficient IFS coding can be applied with compressed data. Proposed block matching with search window subsampling can remarkably reduce the computational complexity with $6.3 \%$.

\section{Block Transform}

The maximal domain pool corresponding to a reference block of size B X B can be thought of as all image blocks of size D X D (D > B) located anywhere in the image to encode. It is typically very large, but it can be trimmed and organized in order to make the search for an optimal domain block tractable.

An initial domain pool $\mathrm{D}$ can be obtained by sliding a window of size $\mathrm{D} X \mathrm{D}(\mathrm{D}=2 \mathrm{~B}$ is used) across the original image. The window is first located with its bottom 'left comer. It then moves from one position to the next by steps of either pixels horizontally to the right, or pixels vertically upwards, in such a way that it remains entirely inside the image support, at all times. The steps can typically be chosen equal to B or $\mathrm{B} / 2$. The domain blocks in this pool $\mathrm{D}$ are then classified, based on their perceptual geometric features [4].

We now propose a procedure for the construction of a transformation pool, and for the directed search of the resulting global pool. The quantization of the parameters of the transformations is dictated by storage requirements. The description of a block transformation should be kept as simple as possible in order to obtain low bit rates. Note that the contraction the transformations can be obtained by multiplying together with the elemental transformations which compose them.

Using the reference and destination region, we extract the contract adjust value and brightness with optimal matrix solution such as Approximation $v_{k}(z)=\alpha z+\beta$. Optimal Solution is given by

$$
\left[\begin{array}{cc}
\int g^{2}(A(x, y)) & \int g(A(x, y)) \\
\int g(A(x, y)) & \int 1
\end{array}\right]\left[\begin{array}{c}
\alpha \\
\beta
\end{array}\right]=\left[\begin{array}{c}
\int w_{k}(x, y) \cdot g(A(x, y)) \\
\int w_{k}(x, y)
\end{array}\right] .
$$

\section{IFS parameter extraction}

The encoding of each range block consists of finding the "best pair" domain block within the domain block pool. The resulting IFS code consists of luminance offset and contrast scaling parameters, and location of the "best pair" domain block. The IFS parameter can be used for image indexing with image characteristics. IFS are defined as a geometrical set whose Hausdorff Besicovitch dimension strictly exceeds the topological dimension [5]. They describe non-Euclidean structures that show self similarity at different scales.

Since the domain block pool of the query image is a subset of the domain block pool of a candidate image, the IFS code corresponding to the local domain block pool is different from that corresponding to the global domain block pool. To minimize the dissimilarity in IFS codes, the candidate images are segmented into blocks of different size and the blocks are arranged. IFS codes 
of each block are then obtained by searching the corresponding domain block pool, instead of the domain block pool of the entire candidate image. The gray scale histogram of gray or color pixels is known to have good indexing and retrieval performance with low computational complexity. Luminance offset and the contrast scaling parameter both depend on the best pair domain block, and are strongly correlated.

\section{Simulation Results}

To verify the proposed IFS image coder we use the optimal solution with the brightness and contrast variation.

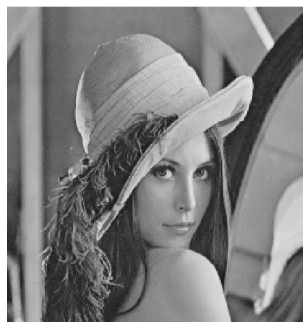

(a)

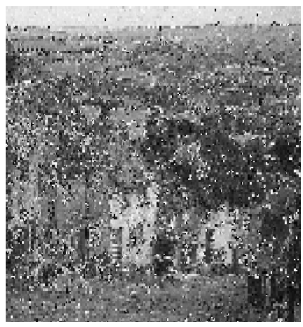

(c)

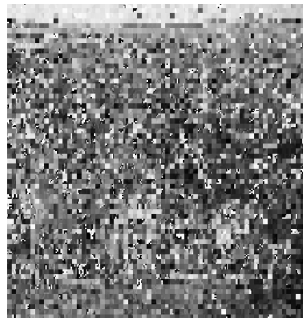

(b)

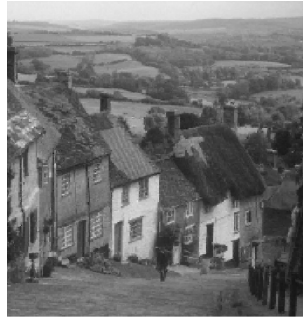

(d)
[Fig. 2] IFS coding images (a) Inital Image

(b) iteration number $=1 \quad(P S N R=12.40 \mathrm{~dB})$

(c) iteration number $=2(\mathrm{PSNR}=14.71 \mathrm{~dB})$

(d) iteration number $=16(\mathrm{PSNR}=32.37 \mathrm{~dB})$

Reference region is extracted using collage error

$\int_{(x, y) \in A}\left[w_{k}(x, y)-\alpha \cdot g(A(x, y))-\beta\right]^{2} d L$

minimization with contrast adjustment value $(\alpha)$ and brightness adjustment value $(\beta)$. The optimal position of reference region, brightness information, and IFS coding mode can be transmitted.

Fig. 2 represents the IFS coding images varying iteration number. Initial image, Fig. 2(a) is transformed by IFS coding parameters which is extracted by minimizing the collage error.

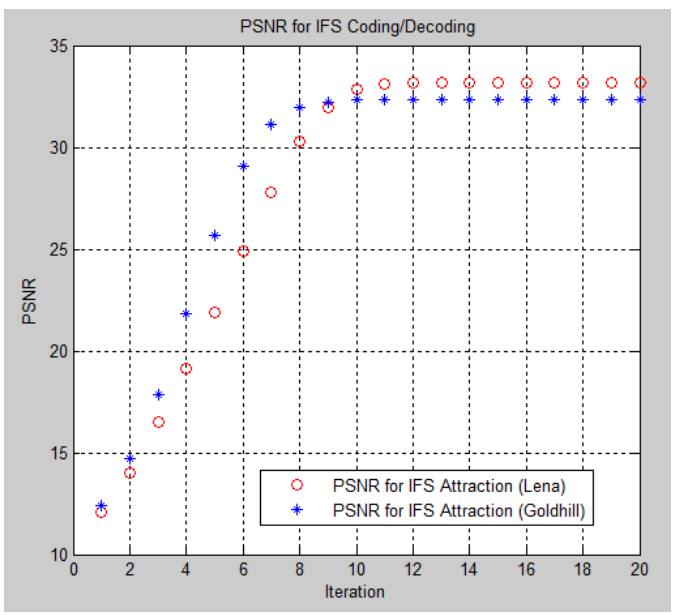

[Fig. 3] PSNR performance for IFS coding

As shown in Fig. 2, The IFS parameters can be employed efficiently for video stream with large frame variation such as shot boundaries.

Fig. 3 shows the PSNR performance for IFS coding for 'Lena' and 'Goldhill' test images, in which the horizontal axis represents the iteration number and the vertical axis shows the PSNR (dB). In Fig. 3, PSNR performance represents stable state when the iteration numbers are more than ten. The image coding using IFS can compress the digital image efficiently with low bit rate using IFS parameters.

PSNR performances for IFS varying block size are shown in [Fig. 4]. As shown in Fig. 4, PSNR performance represents high quality for small $4 \times 4$ block size compare with $8 \times 8$ block. Small block size with $4 \times 4$ need more iteration number for stable state compare with that of $8 \times 8$ block. Coding performance with different input mage represents similar PSNR. 


\section{Conclusions}

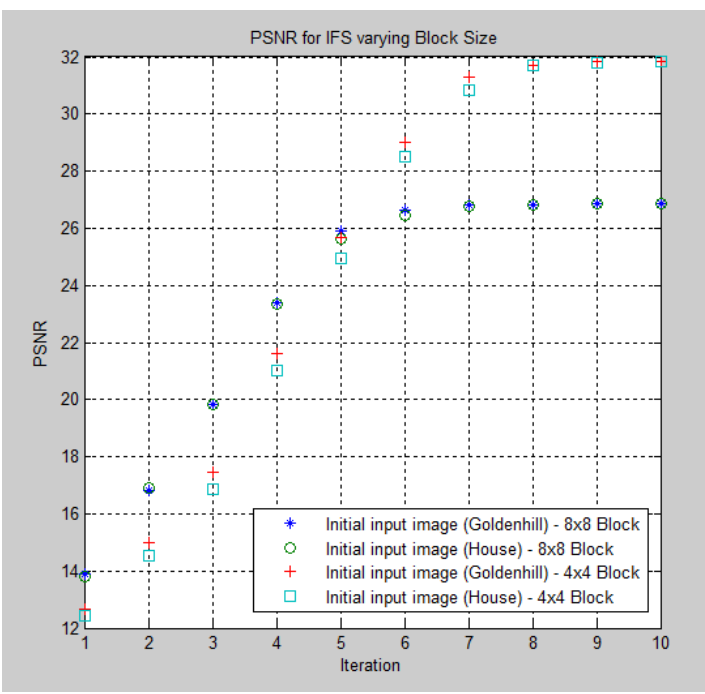

[Fig. 4] PSNR performance for IFS varying block size

We have described the design of digital image coding systems referred to as block coders which are based on a theory of iterated contractive image transformations. The preliminary design issues are to select an adaptive image partition made of nonoverlapping reference cells, to select an interblock distortion measure, and to specify a class of contractive block transformations with quantized parameters. The encoding of an original image then consists of capturing the self-transformability of the original image by searching a global transformation pool for a transformation defined blockwise IFS code under which the image is approximately invariant. A specific block based IFS coding system was presented as well as coding results. The optimized the coding system design to improve the IFS algorithm was presented. The choices in the design a block based IFS coding system affect all of the visual quality of coded images and encoding complexity. Decoding complexity remains fairly low and stable for coding system designs. Future work focuses on the realtime encoding system with complex interframe changes.

\section{References}

[1] J.-H. Jeng, C.-C. Tseng, and J.-G. Hsieh, "Study on Huber fractal image compression," IEEE Trans. Image Processing, vol. 18, no. 5, pp. 995-1003, May 2009.

DOI: http://dx.doi.org/10.1109/TIP.2009.2013080

[2] M. Pi, M. K. Mandal, and A. Basu, "Image retrieval based on histogram of fractal parameters," IEEE Trans. Multimedia, vol. 7, no. 4, pp. 597-605, Aug. 2005. DOI: http://dx.doi.org/10.1109/TMM.2005.846796

[3] Y. Iano, F. S. Silva, and A. Cruz, "A fast and efficient hybrid fractal-wavelet image coder," IEEE Trans. Image Processing, vol. 15, no. 1, pp. 98-105, Jan. 2006. DOI: http://dx.doi.org/10.1109/TIP.2005.860317

[4] M. Pi and H. Li, "Fractal indexing with joint statistical properties and its application in texture image retrieval," IET Image Processing, vol. 2, no. 4, pp. 218-230, Aug. 2008.

DOI: http://dx.doi.org/10.1049/iet-ipr:20070055

[5] M. R. Faraji and X. Qi, "Face recognition under varying illumination with logarithmic fractal analysis," IEEE Signal Processing Letters, vol. 21, no. 12, pp. 14571461, Dec. 2014.

DOI: http://dx.doi.org/10.1109/LSP.2014.2343213

\section{Sang Hyun Kim}

[Regular Member]

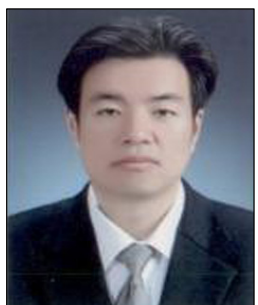

- Feb. 2003 : Department of Electronic Engineering, Sogang University, $\mathrm{PhD}$.

- Mar. 2003 May 2004 : Digital Media Research Laboratory, LG Electronics Inc., Senior Research Engineer. - Jun. 2004 Feb. 2005 :

Computing Laboratory, Samsung Advanced Institute of Technology, Senior Research Member.

- Mar. $2005 \sim$ current : Department of Electronic Engineering, Kyungpook National University, Associate Professor.

$<$ Research Interests> Image Coding, Video Indexing, Computer Vision 